\title{
Performance of PCC-OFDM with Overlapping Symbol Periods in a Multipath Channel $^{\star}$
}

\author{
Jean Armstrong*, Tariq Gill* and Chintha Tellambura $†$ \\ * Department of Electronic Engineering, La Trobe University, Bundoora, Victoria 3083, Australia \\ Email: j.armstrong@ee.latrobe.edu.au \\ $\uparrow$ School of Computer Science, Monash University, Clayton, Victoria 3168, Australia \\ Email: chintha@csse.monash.edu.au
}

\begin{abstract}
Polynomial cancellation coded orthogonal frequency division multiplexing (PCC-OFDM) with overlapping symbol periods is a modulation technique which overcomes many of the disadvantages of OFDM. PCC-OFDM is much less sensitive to frequency offset and Doppler spread than OFDM. Only the length of the equalizer used limits the delay spread that can be tolerated in a PCC-OFDM system. In this paper expressions are calculated for the intercarrier interference (ICI) and intersymbol interference (ISI) caused by timing errors in PCCOFDM. Results are presented for simulations of PCC-OFDM in channels subject to frequency error and multipath. It is shown that good performance can be achieved using equalizers of only moderate complexity.
\end{abstract}

\section{BACKGROUND ON POLYNOMIAL CANCELLATION CODING}

Polynomial cancellation coding (PCC) is a coding technique for orthogonal frequency division multiplexing (OFDM) in which the data to be transmitted is mapped onto weighted groups of subcarriers rather than individual subcarriers. PCC-OFDM has been shown to be much less sensitive than OFDM to frequency offset and Doppler spread $[1,2,3]$. In its simplest form the spectral efficiency of PCCOFDM is approximately half that of OFDM. It has previously been shown $[4,5]$ that PCC-OFDM can be used without any loss in spectral efficiency if intersymbol interference is deliberately introduced at the transmitter by overlapping the symbol periods and the data is recovered at the receiver using an equalizer. In this paper, results of simulations of this system in a multipath channel are presented. Expressions are derived for the ICI and ISI properties of PCC-OFDM.

Fig. 1 shows the transmitter and receiver for a PCC-OFDM system. In OFDM the data to be transmitted in the $i$-th symbol period is mapped directly onto the $N$ inputs $a_{0,} \cdots a_{N-1,}$ of the transmitter IFFT. At the receiver the data is recovered from the FFT outputs $z_{0,1} \cdots z_{N+1}$. In PCCOFDM the data is instead mapped onto weighted groups of subcarriers. The mapping can be onto groups of any size [1] however in this paper mapping onto groups of two will be considered. This reduces the intercarrier interference due to frequency errors by $15-20 \mathrm{~dB}$ compared with OFDM and is sufficient for most applications.

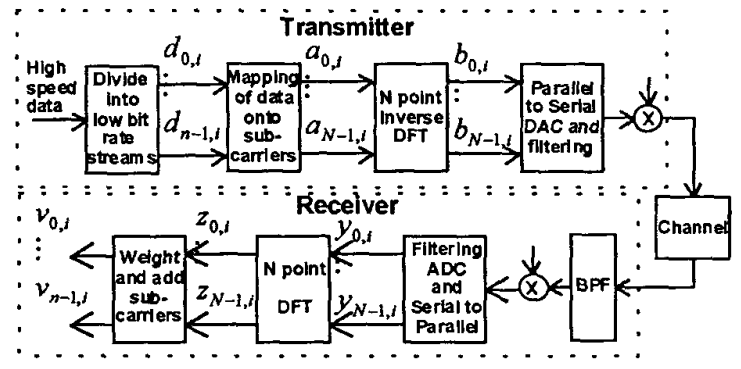

Fig. 1. Block diagram of PCC-OFDM system

In this case, the pair of subcarriers have relative weighting $+1,-1$. Thus, for example, if $d_{i, d}$ is to be transmitted on the adjacent pair of subcarriers $a_{i,}$ and $a_{l+1,}$ then $a_{i, 1}=d_{r,}$, and $a_{i=1,}=-d_{r, 1}$. The mapping of data onto pairs of subcarriers means that the ICI caused by one subcarrier is substantially cancelled by the ICI caused by the other subcarrier in the pair. The groups of subcarriers are combined in the receiver by weighting and adding using the same weightings. In this case $v_{r d}=\left(z_{i, s}-z_{l+1,1}\right) / 2$. This results in the receiver being matched to the PCC waveforms and also contributes to the ICI cancellation properties of the technique. This is because the residual ICI in the received pair tends to cancel [3]. In the rest of the paper the term 'subchannels' will be used to describe the weighted subcarrier pairs in PCC-OFDM.

\section{ICI AND ISI CALCULATION FOR PCC-OFDM}

The data can be recovered from the overlapped symbols in PCC-OFDM with overlapping symbol periods because of the ICI and ISI properties of PCC OFDM. The ICI and ISI caused by misalignment of the receiver FFT window with the received symbol will be analyzed in detail.

Fig. 2 shows the situation being considered. The samples output from the transmitter IFFT in the $i$-th symbol period are given by $b_{0,} \cdots b_{N-1,}$. The samples input to the receiver FFT for the same symbol are $y_{0,} \cdots y_{N-1,}$. In the ideal case for OFDM there is no timing offset and no distortion and these

\footnotetext{
"This work was funded in part by the Australian Research Council
} 
vectors are identical. However we will consider the case where there is no distortion in transmission but where the receiver window is offset by time $\tau$, where $\tau=p T / N, T$ is the symbol period, and $p$ is an integer. (The analysis can be generalized for non-integral $p$ but this requires some assumptions about the transmitter and receiver filtering.)

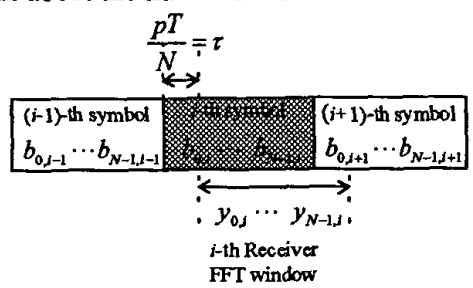

Fig. 2. Timing offset between receiver FFT window and received symbol period

Consider the effect of the $i$-th received symbol in the $i$-th FFT window. Then $y_{k, 1}$ the component of the $k$-th input to the $i$-th receiver FFT due to the $i$-th received symbol is given by

$$
y_{k, \mu}=b_{k+p i} \quad 0 \leq k \leq N-1-p
$$

Then from the definition of the DFT,

$$
\begin{aligned}
z_{m, j} & =\sum_{k=0}^{N-1} y_{k, j} \exp \left(\frac{-j 2 \pi m k}{N}\right) \\
& =\sum_{k=0}^{N-1-p} b_{k+p, d} \exp \left(\frac{-j 2 \pi m k}{N}\right)
\end{aligned}
$$

Using the definition of the IDFT

$$
b_{n, t}=\frac{1}{N} \sum_{l=0}^{N-1} a_{t, d} \exp \left(\frac{j 2 \pi n}{N}\right)
$$

Substituting this in (2) gives

$$
z_{m \perp \perp}=\frac{1}{N} \sum_{k=0}^{N-i-p} \sum_{k=0}^{N-1} a_{t,} \exp \left(\frac{j 2 \pi l(k+p)}{N}\right) \exp \left(\frac{-j 2 \pi m k}{N}\right)
$$

Then considering only the component of $z_{m, 1}$ due to $a_{1, s}$

$$
\begin{aligned}
& z_{m, l, i, i}=\frac{1}{N} a_{l, i} \sum_{k=0}^{N-1-p} \exp \left(\frac{j 2 \pi l(k+p)}{N}\right) \exp \left(\frac{-j 2 \pi m k}{N}\right) \\
& =\frac{1}{N} a_{l, i} \sum_{k=0}^{N-1-p} \exp \left(\frac{j 2 \pi l k}{N}\right) \exp \left(\frac{j 2 \pi l p}{N}\right) \exp \left(\frac{-j 2 \pi m k}{N}\right) \\
& =\frac{1}{N} a_{l, i} \exp \left(\frac{j 2 \pi l p}{N}\right) \sum_{k=0}^{N-1-p} \exp \left(\frac{j 2 \pi k(l-m)}{N}\right)
\end{aligned}
$$

From (5) it can be seen that $\left|z_{m, s, 1}\right|$ depends only on $(l-m)$ the difference in indices between the input and output subcarriers not on the indices directly. Fig. 3 shows $\left|z_{m, \lambda}\right|$ for $a_{l,}=1$ as a function of normalized timing offset $p / N$ and of $(l-m)$. For no timing offset the wanted subcarrier has unity amplitude and all the other subcarriers have zero amplitude. However even small errors in timing cause substantial ICI, which is significant across many subcarriers. As a result, equalization to reduce ICI is usually considered impractical for OFDM and instead a cyclic prefix is used to eliminate ICI at the cost of reduced spectral efficiency.

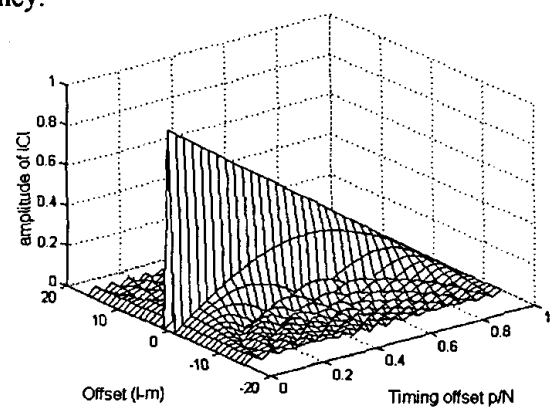

Fig. 3. ICI for OFDM as a function of timing offset and difference in input and output subcarrier index. $N=32$.

For PCC coded data, we are interested in the effect of each input $d_{r,}$ to the transmitter mapping block on each output $v_{x / 1}$ of the weighting and adding block. For simplicity we will consider the case where all subcarriers are used and where $l$ is an even number. $l=2 l^{\prime}$. Similarly $m$ is an even number and $m=2 m^{\prime}$. First the combined contribution of each pair $a_{2 r s}$ and $a_{2 r+1,}$ to each $z_{m s}$ must be found using (5).

$$
\begin{aligned}
z_{m, t+\alpha}= & \frac{1}{N} d_{l^{\prime},} \exp \left(\frac{j 4 \pi l^{\prime} p}{N}\right) \\
& \sum_{k=0}^{N-1-p} \exp \left(\frac{j 2 \pi k\left(2 l^{\prime}-m\right)}{N}\right)\left(1-\exp \left(\frac{j 2 \pi(k+p)}{N}\right)\right)
\end{aligned}
$$

Now by deriving a similar expression for $z_{m+1 / \mu, j}$ and

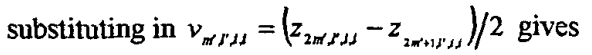

$$
\begin{aligned}
& v_{m^{\prime}, j \Lambda}=\frac{1}{2 N} d_{r,} \exp \left(\frac{j 4 \pi l^{\prime} p}{N}\right) \\
& {\left[\begin{array}{l}
\sum_{k=0}^{N-1-p} \exp \left(\frac{j 4 \pi k\left(l^{\prime}-m^{\prime}\right)}{N}\right)\left(1-\exp \left(\frac{j 2 \pi(k+p)}{N}\right)\right) \\
\left(1-\exp \left(\frac{-j 2 \pi k}{N}\right)\right)
\end{array}\right]}
\end{aligned}
$$

From (7) it can be seen that for PCC the magnitude of the interchannel interference depends only on $\left(l^{\prime}-m^{\prime}\right)$ and $p$, but not on $l^{\prime}$ or $m^{\prime}$ directly.

Fig. 4 shows $\left|v_{m^{\prime}, r^{\prime}, 1}\right|$ as a function of $\left(l^{\prime}-m^{\prime}\right)$ and $p$ for $d_{r, 1}=1$. This shows that in contrast to OFDM, for small timing offsets the interference in all other subchannels is negligible. Even for the worst case timing error of half a symbol period, the interference is only significant in the few immediately adjacent subchannels. As a result the equalizers required for PCC-OFDM with overlapping symbol periods are comparatively simple. 


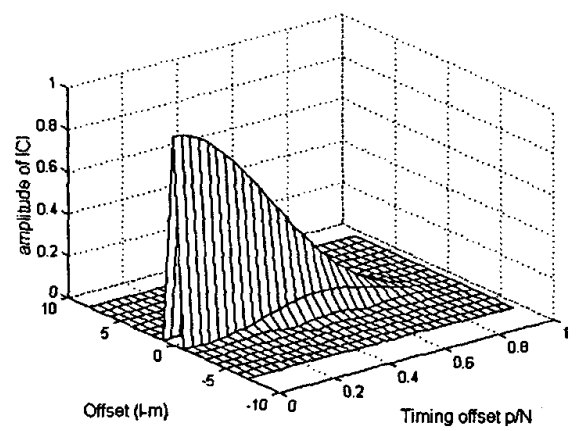

Fig. 4. Interference between subchannels for PCC-OFDM as a function of timing offset and difference in input and output subchannel index. $N=32$.

In PCC-OFDM the energy of each symbol is concentrated in the middle of each symbol period. This can be shown by calculating the component of the transmitter IFFT output, $b_{k, i}$, that is due to input $d_{r, 1}$

$b_{k, l^{\prime}, i, i}=\frac{1}{N} d_{l^{\prime}, i} \exp \left(\frac{j 2 \pi 2 l^{\prime} k}{N}\right)-\frac{1}{N} d_{l^{\prime}, i} \exp \left(\frac{j 2 \pi\left(2 l^{\prime}+1\right) k}{N}\right)$ $=\frac{1}{N} d_{r, i} \exp \left(\frac{j 2 \pi 2 l^{\prime} k}{N}\right)\left(1-\exp \left(\frac{j 2 \pi k}{N}\right)\right)$

This has the form of a complex exponential of frequency $2 \pi d^{\prime} / N$ windowed with the complex windowing function $1-\exp (j 2 \pi k / N)$. Fig. 5 shows this windowing function for $N=64$. For PCC implementations with less symmetry in the mapping of data to subcarriers there is no windowing equivalent, however the energy of each symbol is still concentrated in the middle of cach symbol period.
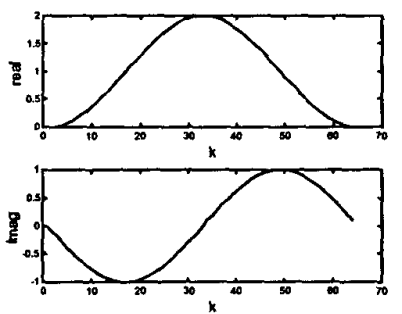

Fig. 5. Complex windowing function

\section{PCC-OFDM WITH OVERLAPPING SYMBOL PERIODS}

Because of the ICI and ISI properties derived in the previous section, PCC-OFDM can be transmitted with overlapping symbol periods and the data recovered in the receiver with little noise enhancement using a comparatively simple equalizer.

Fig. 6 illustrates the basic concept. Intersymbol interference (ISI) is deliberately introduced at the transmitter to increase the data rate $[4,5]$. For $d=T / 2$, where $d$ is the overlap between symbols, the transmitted signal at any instant is the sum of components due to two different symbols. The concept can be generalized to other values of $d$. Fig. 7 shows the form of the receiver required to recover the information from the overlapped symbols.

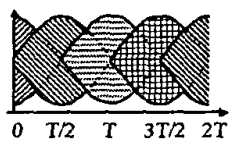

Fig. 6: PCC-OFDM with overlapping in time domain

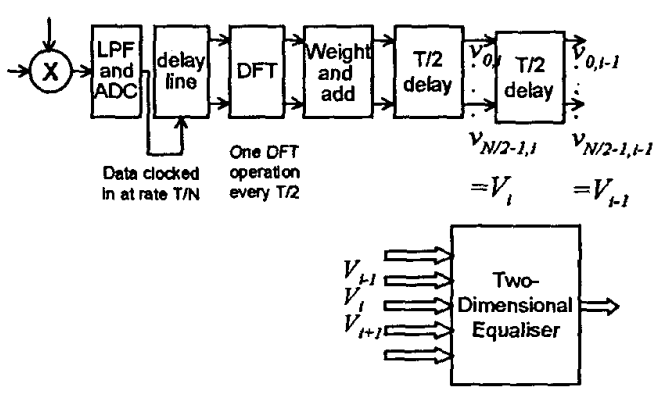

Fig. 7: Receiver for PCC with overlapping symbols

The equalizers required to recover the information from the received signal are two-dimensional: interference occurs in the time domain between symbols and in the frequency domain between subchannels within a symbol. However because of the ICI/ISI properties of PCC there are only a small number of significant tap values. Even severe multipath distortion has little effect on the number of significant terms. All of the equalization techniques used for one dimensional equalization such as zero forcing linear equalization, minimum mean square error (MMSE) equalization, decision feedback equalization (DFE) and maximum likelihood sequence estimation (MLSE) can be extended to two dimensions and applied to OFDM [6-9]. Similar equalizer structures were used in these simulations, but for PCCOFDM the equalizers operate on the output of the weighting and adding block, rather than on the output of the receiver DFT. This both reduces the complexity of the equalizers and contributes to the cancellation properties of the PCC code [3]. The MMSE formula used to calculate the matrix of equalizer taps used in the simulations in this paper is given by

$$
C_{\text {mina }}=\frac{E\left\{d^{2}\right\}}{E\left\{n^{2}\right\}} A H_{s}^{*}\left(\frac{E\left\{d^{2}\right\}}{E\left\{n^{2}\right\}} H_{s} H_{s}^{*}+H_{n} H_{n}^{*}\right)^{-1}
$$

where $H_{s}$ represents the effect of the channel etc on the signal component. $H_{s}$ is a matrix, which depends on the transfer function from the input of the transmitter mapping block to the output of the receiver, weighting and adding block. The dimensions of $H_{s}$ depend on the length of the equalizer. $H_{N}$ represents the effect of overlapping and of the weighting and adding block on the noise. Because of the overlapping, the noise samples at the input to the equalizer 
are not independent. $E\left\{d^{2}\right\}$ is the variance of the data at the input to the transmitter mapping block. $E\left\{n^{2}\right\}$ is the variance of the noise at the input to the receiver. When there is frequency offset between transmitter and receiver, better performance can be achieved by including the effect of ICI due to frequency error in the calculation of MMSE equalizer taps. This was not done in the simulations presented in this paper.

\section{PERFORMANCE OF DFES FOR PCC-OFDM}

In all of the simulations described in this section 4-QAM modulation was used and $N=64$. PCC-OFDM is designed for use in channels subject to frequency errors and multipath, however it has only slightly worse performance than OFDM in AWGN.

Fig. 8 shows the performance of PCC-OFDM in AWGN when the overlap between symbols is $T / 2$ and DFEs of varying complexity are used. In these simulations it is assumed that correct decisions are fed back - there is no error propagation. While this would not be a realistic assumption for single carrier systems, it is realistic for many potential applications of PCC-OFDM where error correcting codes can be used across the subchannels in each symbol, and errors corrected before the decisions are fed back.

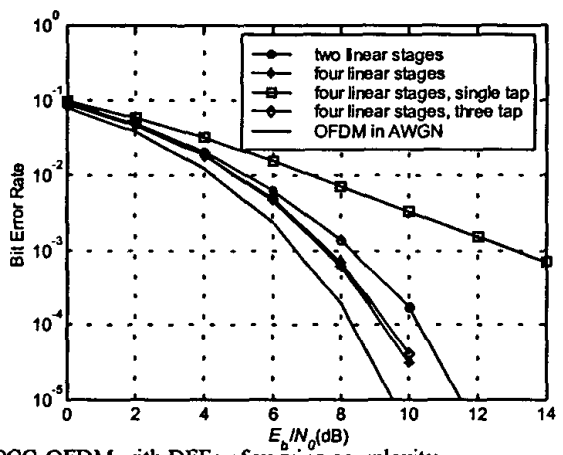

Fig. 8: PCC-OFDM with DFEs of varying complexity.

The first two graphs in Fig. 8 show the results for equalizers with decision feedback and two and four linear feedforward stages when all tap values across each symbol are used, even very small ones. For comparison the results for OFDM in AWGN are also included. The equalizer with four linear stages gives performance within $\mathrm{a} \mathrm{dB}$ of that of OFDM. Fig. 8 also shows the effect of simplifying the equalizer by using for each subchannel only the main tap and immediately adjacent taps. When only a single tap is used in each stage, considerable degradation occurs, however using only three taps, rather than $N / 2$ taps, in each stage gives negligible degradation.

Fig. 9 and 10 show the effect of frequency offset on PCCOFDM and OFDM. Fig. 9 shows the signal to noise ratio at the equalizer output for PCC-OFDM and at the FFT output of OFDM, as a function of normalized frequency offset $\Delta f T$.
Results are shown for two values of $E_{b} / N_{0}$. For the case of OFDM, the total noise is a combination of additive white Gaussian noise and ICI due to frequency error. For PCCOFDM, residual ISI also contributes. For $\Delta f T>0.1$, PCCOFDM has a lower SNR than OFDM, for both values of $E_{b} / N_{0}$. This is because the ICI due to frequency offset is the dominant impairment. For very small $\Delta f T$, the SNR of OFDM is lower, because the residual ISI and the noise enhancement of the PCC-OFDM equalizer become relatively more significant. If necessary, residual ISI can be reduced by increasing the length of the equalizer. At intermediate values of $\Delta f T$, the relative performance of the two systems depends on the value of $E_{b} / N_{0}$. In practice, the benefits of using PCC-OFDM are likely to be greater than these results suggest, as the elimination of the cyclic prefix may allow a smaller value of $N$ to be used for PCC-OFDM than OFDM.

Similar results can also be obtained for channels subject to Doppler spread because PCC-OFDM is inherently less sensitive to frequency errors. Fig. 10 shows the effect of frequency error on the BER for $E_{b} / N_{0}=10 \mathrm{db}$.

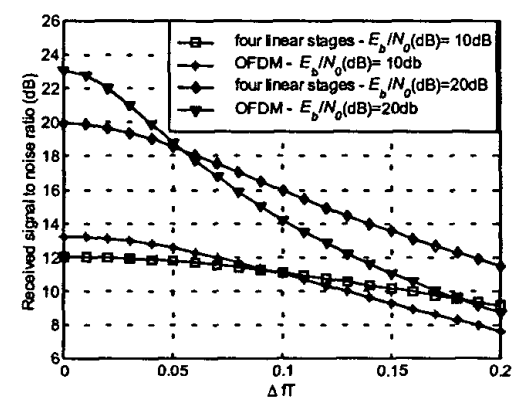

Fig. 9: Signal to noise ratio at equalizer output of PCC-OFDM and at FFT output for OFDM as a function of frequency error

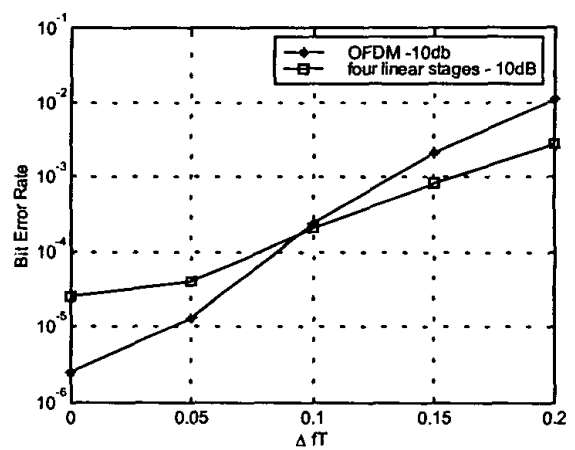

Fig. 10: Performance of OFDM and PCC-OFDM in systems subject to frequency error.

Fig. 11 shows the performance of PCC-OFDM in a multipath channel. In this case there are two paths, the second path has power 0.75 of the first path. The receiver is synchronized to the first path. The multipath causes severe 
frequency selective fading which results in very high bit error rates in some subcarriers in OFDM and some subchannels in PCC-OFDM. The errors in these bad subcarriers and subchannels dominate the results. Fig. 11 shows the effect of varying the delay of the second path relative to the first path. For OFDM there is little effect, until the delay exceeds the length of the cyclic prefix. In these simulations a cyclic prefix of six samples was used. Once the delay exceeds the length of the cyclic prefix, the BER increases rapidly, particularly for the case of $E_{b} / N_{0}=15 \mathrm{~dB}$. In contrast the performance of PCC-OFDM improves as the delay increases. This is because the shaping of PCC-OFDM symbols means that as the relative delay between paths increases the interference caused in a symbol by the delayed replica of itself is reduced. The ISI in the following symbol is corrected by the DFE. The amount of delay spread which can be tolerated in the PCC-OFDM system depends on the design of the equalizer in the receiver, in contrast to OFDM where this is determined by the length of the cyclic prefix. In the multipath channel most of the errors are due to the few bad subcarriers or subchannels. The performance can be substantially improved by the use of suitably designed error correcting codes or by using spread spectrum techniques such as multicarrier code division multiple access (MC-CDMA).

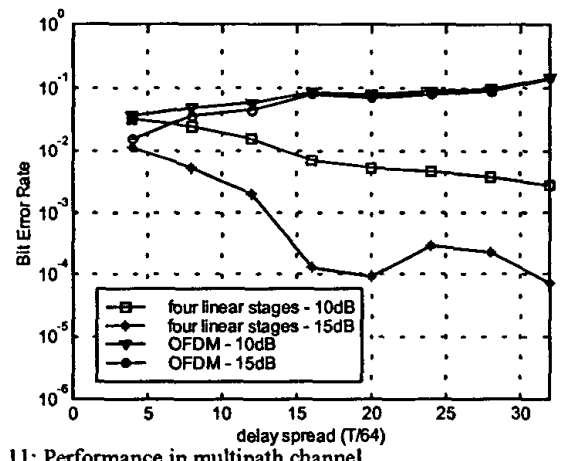

Fig. 11: Performance in multipath channel

\section{PERFORMANCE OF LINEAR EQUALIZERS FOR PCC-OFDM}

In the previous section it was shown that PCC-OFDM gives excellent results when a DFE is used. However the use of decision feedback limits the ways in which PCC-OFDM can be combined with error coding and multiple access techniques. Linear equalization can be used in a more flexible way. Fig. 12 shows the performance of linear equalizers of varying length and complexity. The effect of varying the overlap between symbols is also shown.

For these equalizers, $n$ is the total number of linear stages. Equal numbers of preceding and following symbols were used as inputs to the equalizers. For $d=T / 2$ linear equalization causes considerable noise enhancement. Reducing the overlap to $27 T / 64$ reduces this effect substantially. The degradation in performance is negligible if only three taps per subchannel are used in each stage. PCCOFDM systems with linear equalization are also very insensitive to frequency errors and multipath distortion [10].

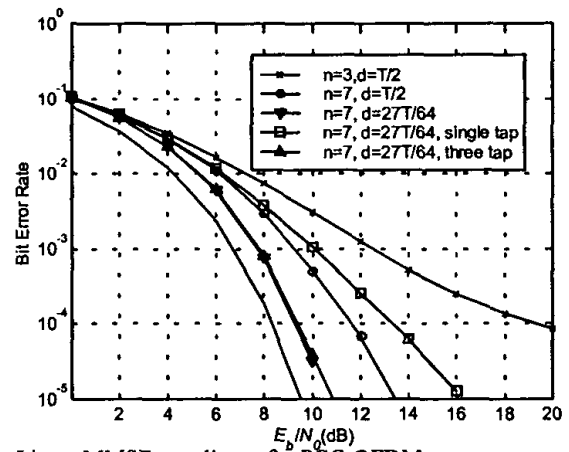

Fig. 12: Linear MMSE equalizers for PCC-OFDM

\section{vi. CONCLUSIONS}

In this paper the ICI and ISI properties of PCC-OFDM have been analyzed. Simulation results have been presented which show that PCC-OFDM has a much lower BER than OFDM in channels subject to multipath distortion with long delay spread and that the ICI due to frequency error is lower in PCC-OFDM than OFDM. It has been shown that, although the equalizers required for PCC-OFDM are two dimensional, these are of only moderate complexity as most tap values can be set to zero with little degradation in performance.

\section{REFERENCES}

[1] J. Armstrong "Analysis of new and existing methods of reducing intercarrier interference due to carrier frequency offset in OFDM", IEEE Trans. Commun., vol. 47, pp365-369, March 1999.

[2] J. Armstrong, P.M. Grant, G. Povey, "Polynomial cancellation coding of OFDM to reduce Intercarrier interference due to Doppler spread", IEEE Globecom, vol 5, pp. 2771-2776, 1998.

[3] K. Seaton and J. Armstrong, 'Polynomial Cancellation Coding and finite differences', IEEE Trans. Inf. Theory, vol 46, nol, pp.311-313 Jan. 2000.

[4] J. Armstrong, J. Shentu and C. Tellambura "Frequency Domain Equalization for OFDM systems with mapping of data onto subcarrier pairs and overlapping symbol periods", "Coding, Communications and Broadcasting", pp. 217-228. edited by P.G. Farrell, Mike Damell and Bahram Honary, to be published March 2000, R.S.P.

[5] J. Armstrong, "Data transmission and reception in multicarrier modulation systems", PCT application WO9962207.

[6] K. Matheus et al., "Two Dimensional (recursive) channel equalization for multicarrier systems with soft impulse shaping (MCISS)", in IEEE Globecom conf, Sydney, November 1998, pp956-961.

[7] L. Vandendorpe, J. Louveaux, B. Maison and A. Chevreuil, "About the asymptotic performance of MMSE MIMO DFE for filter-bank based multicarrier transmission", JEEE Trans. on Communications, vol. 47, pp. 1472-1475.

[8] L. Vandendorpe and $O$. Van de Weil, "Performance analysis of linear MIMO equalizers for multitone DS/SS systems in multipath channels", Wireless Personal Communications, vol. 2, 1995, pp145-165.

[9] V.Demoulin and M. Pecot, "Vector Equalization: an alternative approach for OFDM systems", Ann. Telecommun., vol. 52, no, 1-2, 1997, pp. 4-11.

[10] J. Armstrong and C. Tellambura, "Multicarrier CDMA systems using PCC-OFDM" IEEE Vehicular Technology Conference, May 2000. 